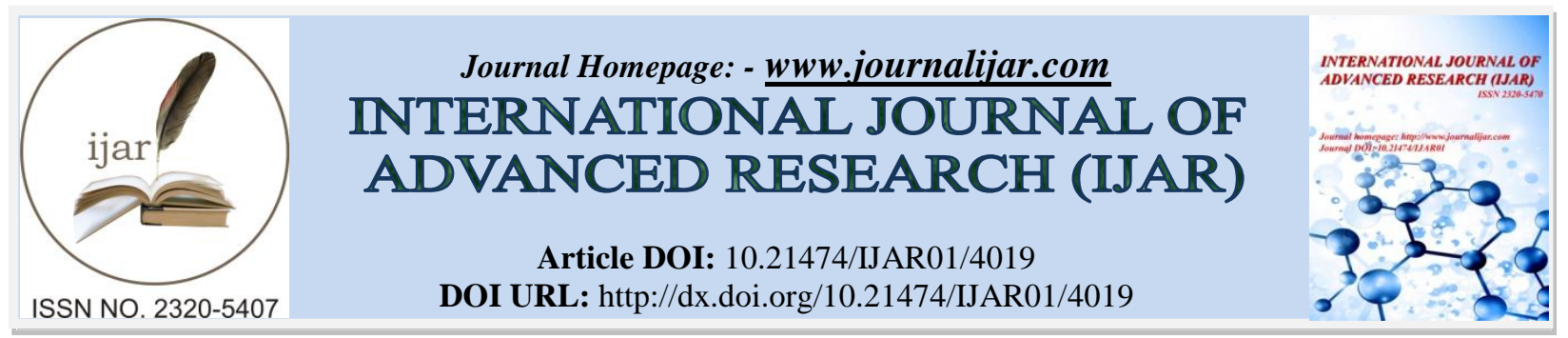

RESEARCH ARTICLE

\title{
UNDERSTANDING BETWEEN MEDICAL AND DENTAL PROFESSIONALS.
}

Nurul Syamimi binti Mohd Azlan Sunil ${ }^{1}$ and "Dr. Brigit Eapen².

1. Bachelor of Dental Surgery (year 1), Saveetha Dental College, Chennai-600077.

2. Professor, Department of Oral Surgery, Saveetha Dental College and Hospitals, No.162, PH road, Chennai600077 .

\section{Manuscript Info}

Manuscript History

Received: 23 February 2017

Final Accepted: 19 March 2017

Published: April 2017

Key words:-

Medical-dental understanding; Attitude; Awareness; Dentistry; Interprofessional relationship

\section{Abstract}

Aim and objective: The aim of this survey is to find out the attitude of medical professionals to the field of dentistry. Thereby creating an awareness of the developing good rapport between medical professionals and dentist and also encouraging dental professionals to be equally aware of the importance of the medical field and also be equally competent and discipline in their approach. Background: Various dental professionals come across unhealthy professional egoism and criticism from medical professionals in their daily practice. Also, many medical professionals are unaware of the scope of dentistry and thereby encroach into their field of interest, thus, creating an unhealthy professional relationship. Reason: The reason this survey was conducted is to make aware of the importance of dentistry in medical sciences. It is essential to understand the knowledge and understanding of all dental profession within the medical sector to ensure a better care for patients as a whole. Materials and Methods: A survey was done to find out the attitude of medical professionals to the field of dentistry. A pretested questionnaire containing 10 questions on personal experiences of dentists dealing with medical professionals was prepared for the dental professionals to be filled up. 100 dental professionals, both male and female from Saveetha Dental College were assessed through the questionnaire. The questionnaires were directly distributed to the subjects and finished questionnaires were immediately collected on site. The subjects are between the age of 23 to 59 years old. Results: A total of 100 questionnaires were distributed and $100(100 \%)$ were returned.Based on the survey, the level of understanding and attitude between medical and dental professionals were positive towards each other. Most of the subjects agree that their relationship with medical doctors are in good rapport and are stable. Conclusion: The findings from this survey showed that the subjects have established good rapport with their medical acquaintances despite the differences in their field of career.

Copy Right, IJAR, 2017,. All rights reserved. 


\section{Introduction:-}

Dental practitioners and medical doctors have their patients' wellbeing secured head to toe, yet they advance health in various ways. While dental specialists concentrate on oral health, specialists treat disease and harm in other physiological system. Their range of focus isn't the main contrast: They take after separate educational paths, and dental practitioners have less specialty alternatives than doctors. What both offer, however, is a brilliant occupation viewpoint or above-average pay[1].

They likewise endorse medications, request diagnostic tests, diagnose afflictions, and record patient data[2]. Doctors of medicine regularly have a specialization, for example, general practice, gynaecology, dermatology, paediatric drug, orthopaedics, or numerous others. All surgeons are medicinal specialists.

Medical doctors' employments change by forte and workplace. While emergency room doctors will work in quick pace, high-stretch situations, numerous family specialists can set their own schedules in a private medicinal practice. Peopling in sickness and harm can be extremely compensating, yet the employment can be distressing and convey an enthusiastic toll, also.

Another important branch of medicine is dentistry. It is included in the study, diagnosis, aversion, and treatment of diseases, disorders and states of the oral depression, regularly in the dentition additionally the oral mucosa, and of adjoining and related structures and tissues, especially in the maxillofacial (jaw and facial) area [3].It is thought that dental surgery was the first specialization from medicine[4].

Dental practitioners' areas of consideration incorporate their patients' teeth and gums as well as the muscles of the head, neck and jaw, the tongue, salivary organs, the nervoussystem of the head and neck and different ranges[5]. During a comprehensive exam, dental practitioners inspect the teeth and gums, however they likewise search for lumps, swellings, discolorations, ulcerations - any variation from the norm. Whenever fitting, they perform procedures, for example, biopsies, diagnostic tests for infectious or chronic disease, salivary glandfunction, and screening tests for oral tumour.Oral diseases are major public health problems due to their high incidence and prevalence across the globe with the disadvantaged affected more than other socio-economic groups [6].

Furthermore, dental practitioners can spot early cautioning signs in the mouth that may indicate illness somewhere else in the body. Dental specialists' preparation additionally empowers them to perceive circumstances that warrant referring patients for care by dental specialists or doctors.

Various dental professionals come across unhealthy professional egoism and criticism from medical professionals in their daily practice. Also, many medical professionals are unaware of the scope of dentistry and thereby encroach into their field of interest, thus, creating an unhealthy professional relationship.

This survey is important in order to understand the attitude of medical professionals to the field of dentistry and make aware of the importance of dentistry in medical science.

\section{Materials and Methods:-}

A pretested questionnaire containing 10 questions on personal experiences of dentists dealing with medical professionals was prepared for the dental professionals to be filled up. 100 dental professionals, both male and female from Saveetha Dental College were assessed through the questionnaire. The questionnaires were directly distributed to the subjects and finished questionnaires were immediately collected on site. The subjects are between the age of 23 to 59 years old.

The questionnaire includes age and sex of the subjects, to their profession, relationship with medical colleagues and their opinion and ideas on how to improve the understanding between medical and dental professionals. 
Date:

\section{UNDERSTANDING BETWEEN MEDICAL AND DENTAL PROFESSIONALS} SURVEY AMONG PROFESSIONALS IN SAVEETHA UNIVERSITY

Dear participants (Sir/Ma'am/Ms),

This questionnaire is part of the Behavioural Science research project. Please answer by ticking one alternative for each question or by writing your or by writing your comments below the questions. Your participation is highly appreciated!

Age:

Gender:

1. Are you a/an

a) academician b) practitioner c) consultant

2. Are you associated with any medical colleagues in your practice or profession?

Yes / No

3. Do you find any difficulty in communicating with a medical professional?

Yes / No

4. Do you think a medical professional is aware of the scope of a dental professional?

Yes / No / Maybe

5. Do you feel any change in attitude in your colleagues in the medical fraternity towards your profession?

Yes / No / Maybe

6. If so, what could be the possible reason?

Comment:

7. Do you consider a dental professional as equal to a medical professional for service to a patient?

Yes / No

8. Do you feel professionals in the field of medicine encroach into your field of expertise?

Yes / No

9. Do you feel the rapport between medical and dental can be improved right from the training periods itself?

Yes / No

10. What would you think could improve the understanding between medical and dental professionals? Comment: 


\section{Results:-}

A total of 100 dental professionals in Saveetha Dental College participated in this study. The response rate of participants was 100\%. Among the participants, $43 \%$ were female and 57\% were male, aged 23 to 59 years. The results were gathered through the questionnaires and they were as follows.

In question one, the participants were asked whether they are academicians, practitioners or consultants. 18\% claimed to be academicians only, $7 \%$ are practitioners only and $11 \%$ are consultants only while $18 \%$ are both academicians and practitioners, $8 \%$ are academicians and consultants, $13 \%$ are practitioners and consultants, and the remaining and highest percentage are of altogether academician, practitioner and consultant, which is $26 \%$.

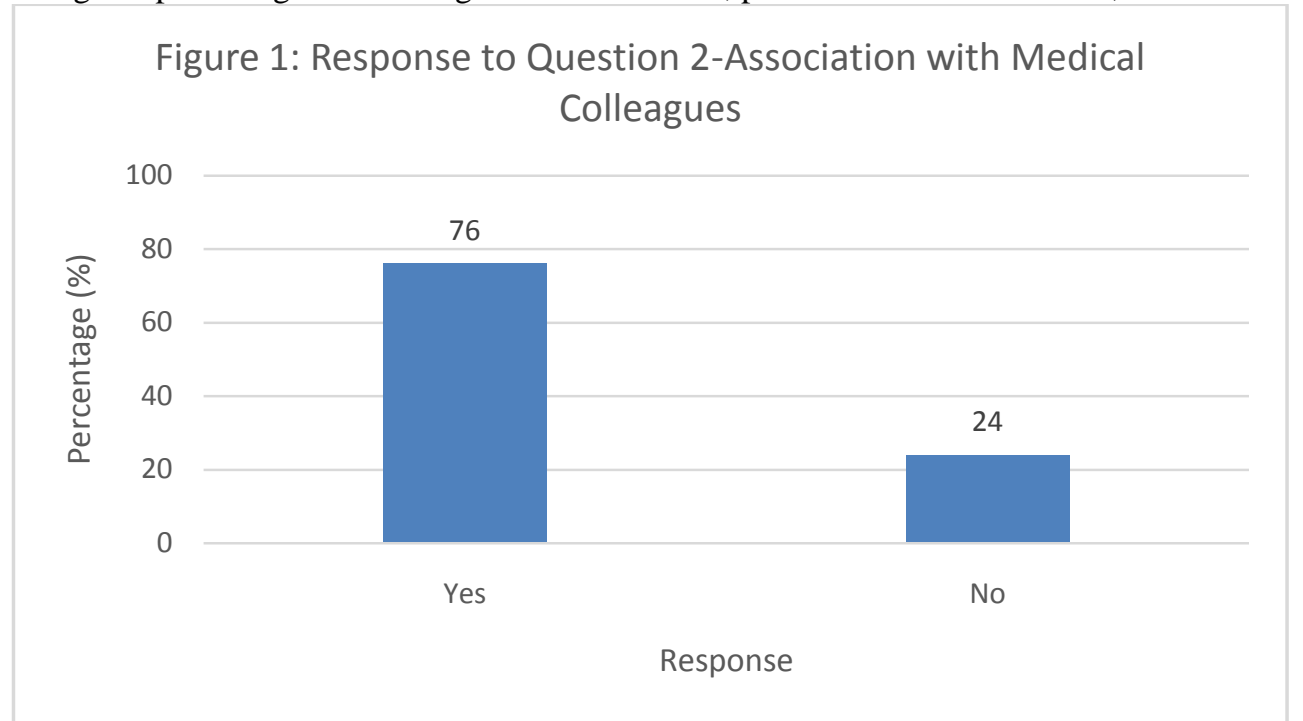

Figure 1:- shows the result of the second question, which is whether the participants are associated with any medical colleagues in their practice or profession. From the figure, it shows that $76 \%$ answered yes and $24 \%$ answered no. This shows that most dental professionals do have the experience of working closely with medical professionals while a minor number do not.

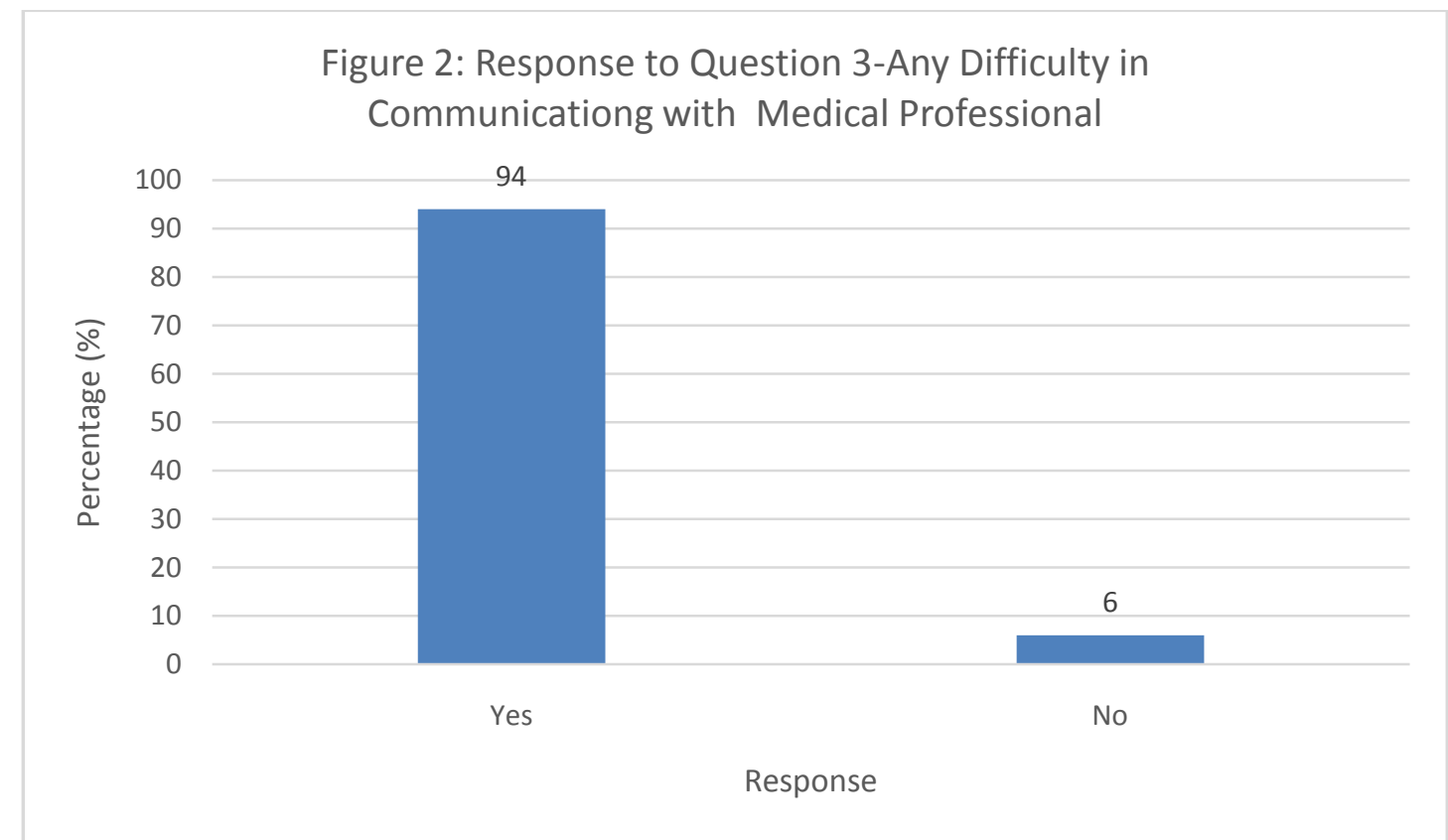

Figure 2:- shows the participants' respond to question 3, which is whether they have any difficulty in communicating with a medical professional. According to figure 2 , only $6 \%$ of the participants have difficulty in conversing with medical doctors while a major percentage of 94 do not face this problem. 
When asked whether they think medical professionals are aware of the scope of dentistry, exactly $44 \%$ agreed on medical professionals' awareness on the scope of dental professionals. Contrary to that, $28 \%$ answered no while the remaining $28 \%$ answered maybe. This is shown in Figure 3.
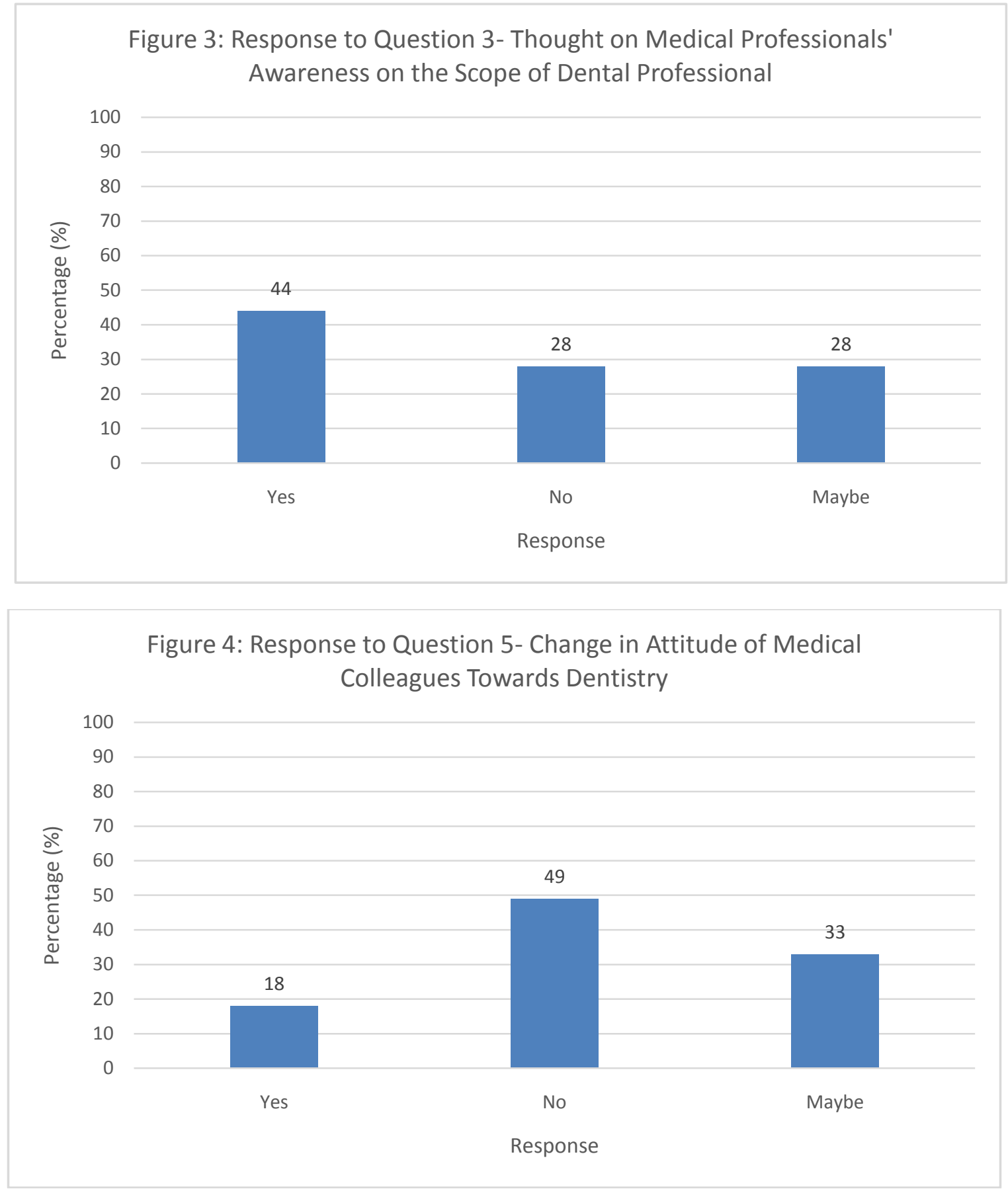

Respond to Question 5 as shown in Figure 4 portray $18 \%$ of the participants agreed there are changes in attitudes of their colleagues in the medical fraternity towards their profession. Most of the participants disagree with the statement, with the percentage of $49 \% .33 \%$ are somewhat in between.

Question 6 asked on their opinion as to what could be the possible reason for the change for those who answered (a) and (c) which are yes and maybe respectively. Most of them gave common opinions. Some of opinions given is the sense of superiority of medical doctors as their profession involves the overall health and major part of human body 
of an individual. Other thoughts are some medical doctors do not understand that dentists deal with overall head and neck region and not the oral cavity alone. Despite both medical and dental doctors share a common above-average pay, egoism continues to show-off among medical doctors toward dentists as they feel more superior and knowledgeable, as one of the answers suggest. On a different viewpoint, a majority (87\%) of those who answered yes and maybe for the previous question, believe that there is a positive change in the attitude of their medical colleagues towards dentistry as some cases are in need of discussion and teamwork from both sides.

When asked whether dental professionals consider themselves as equal to a medical professional for service to a patient in Question 7, $21 \%$ circled no while the remaining (79\%) responded yes, as shown in Figure 5.

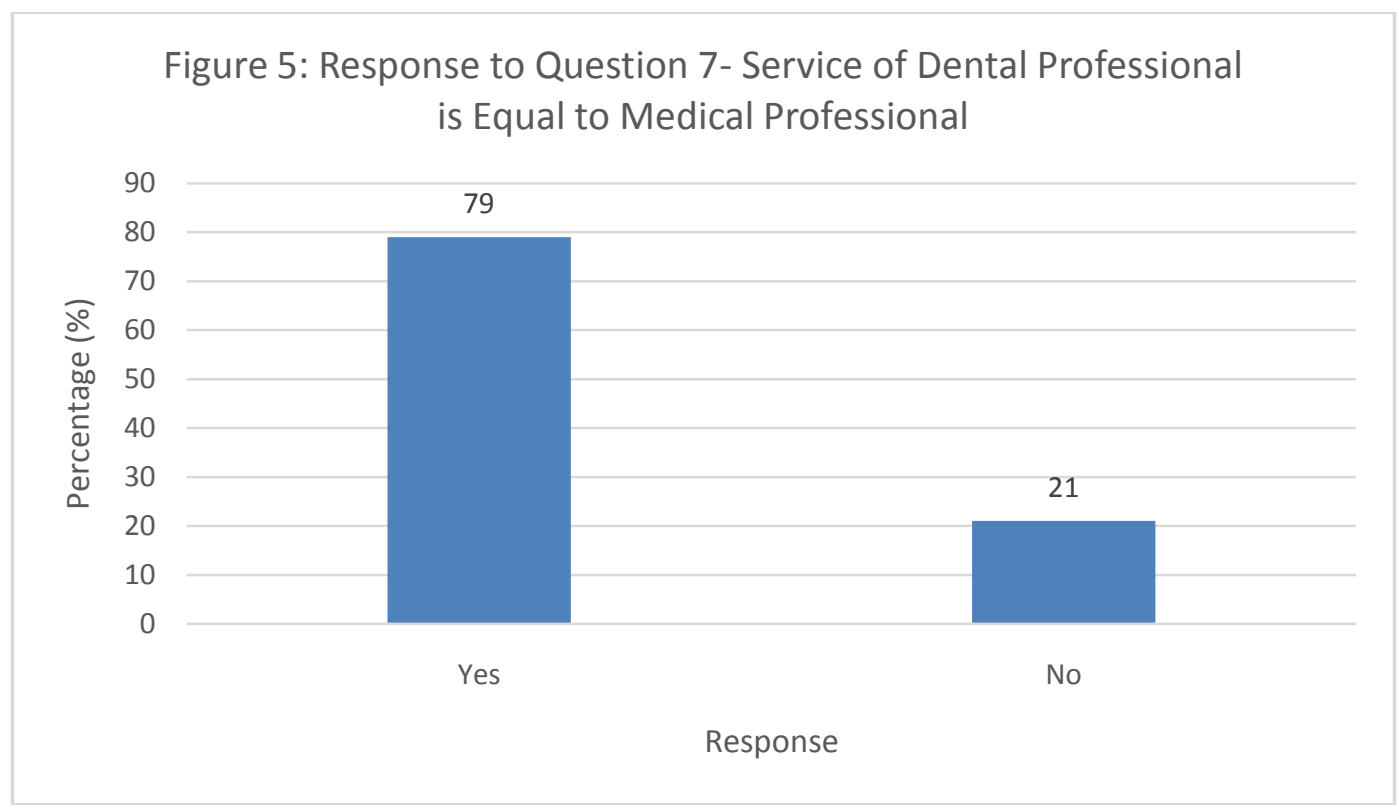

Figure 6:- shows the percentage of yes and no answer to Question 8. In Question 8, participants were asked if they feel professionals in the field of medicine encroach into their field of expertise. Results from the survey showed that $47 \%$ of the participants agreed on answering yes, while $57 \%$ disagree with the fact that medical doctors encroach into their field of expertise.

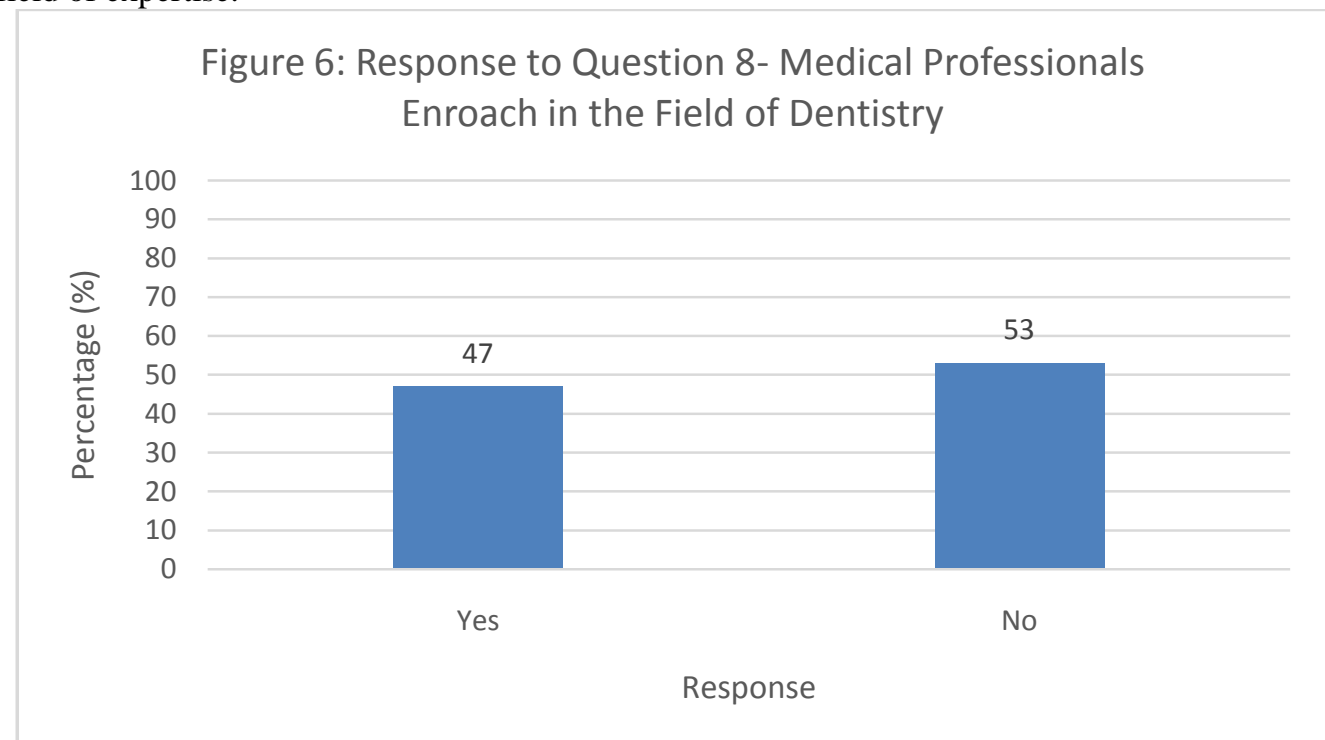

When asked if they feel the rapport between medical and dental doctors can be improved right from the training periods itself in Question 9, a majority of the participants, exactly 94\% have given a yes to the question. The remaining 6\% went with a no. Percentage of response to Question 9 is shown in Figure 7. 


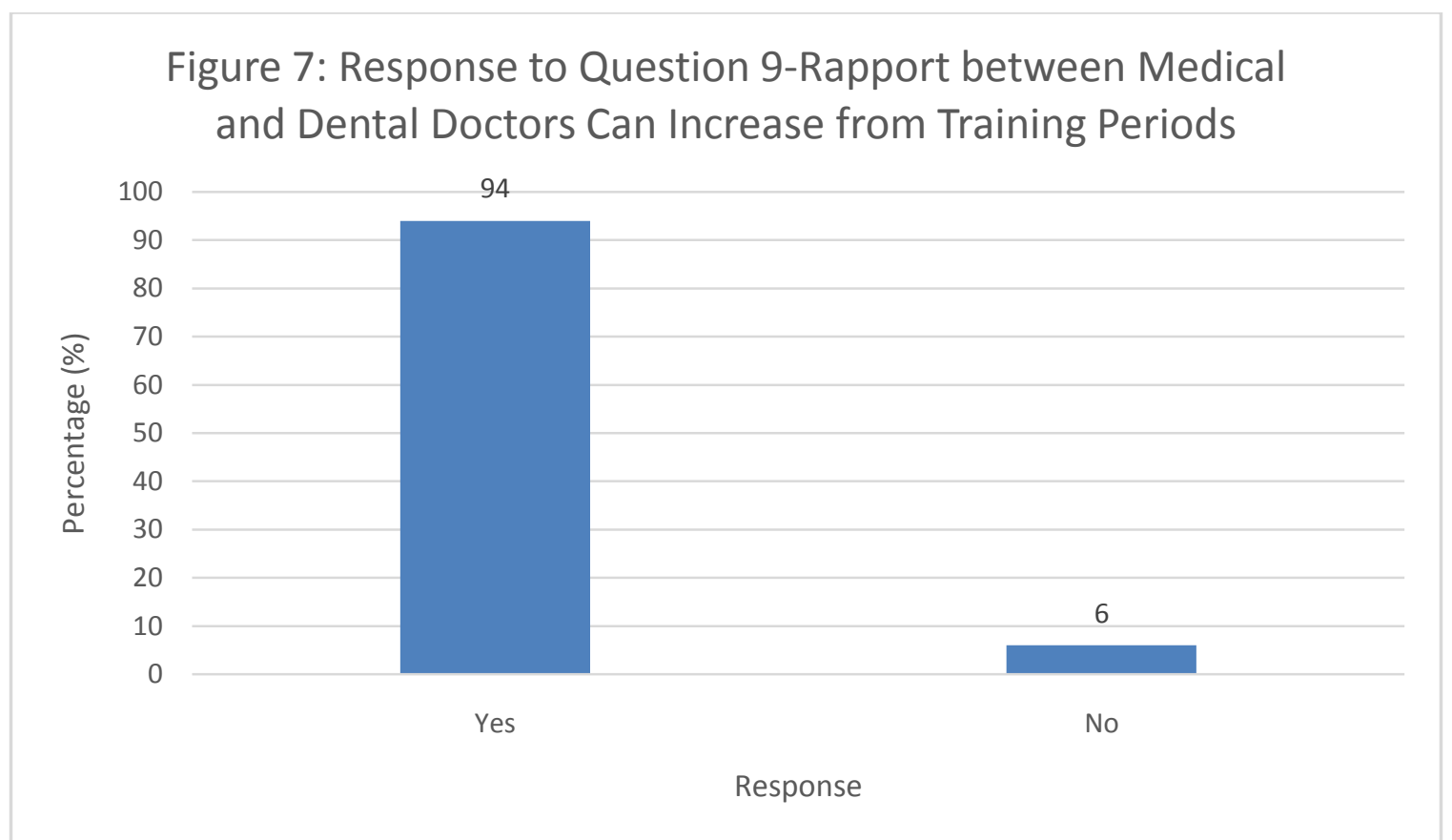

Question 10 requires the participants to give their opinions and ideas on improving understanding between medical and dental professionals. Their comments include:

- Discussing cases that require teamwork from both sides

- Make first year of study common for both

- Yearly conference and seminars with special cases to share ideas

- Include dental studies in medical colleges

- Explain the importance of dentistry

- Organizing joint case discussions

- Make aware the effect of oral health to overall systemic health

\section{Discussion:-}

The results of this study show that most of the interviewed professionals are aware of their relationship with medical professionals and understand the importance of keeping a good rapport. There are many ways to keep the rapport between medical and dental professionals, one of the most effective is by collaboration.

Physicians and dentists rarely have the opportunity to discuss the oral and systemic links. Health care provider sees oral health problems as being separate from general health and should be treated by dentists alone. This goes to show that not all doctors understand the importance of oral hygiene to systemic health. Mouth is clearly part of the body and are connected to other tissue and organs. For instance, more severe periodontal diseases are associated with diabetes mellitus and impaired immunity $[7,8]$ and also teeth erosion can be caused by gastroesophageal reflux [9].

Doctors should also pay attention oral health conditions and work closely with dentists as necessary to ensure holistic health care. A study showed that one of the commonest disease of pre-schoolers in Hong Kong is dental carries that affect more than half of them by the age of 5 years [10]. This is when understanding and knowledge of both medical doctors and dentists become helpful. Paediatricians and doctors should have a good understanding of this important epidemic, give appropriate health advice, and make appropriate early referrals to dentists[11]. The importance of maintaining good oral hygiene and oral health in the prevention of pneumonia among nursing home residents[12], as well as in the management of patients with diabetes[7], cardiovascular diseases[13], and chronic kidney disease [14] have been amply reported.

Oral and systemic health are certainly related. In order to provide high-quality health service, an interdisciplinary and holistic approach should be adopted [15]. Despite the differences in the level of knowledge, work experience 
and income, both medical and dental professionals share a passion and goal to provide health treatment as a whole and not just respective body parts.

\section{Conclusion:-}

The findings from this survey showed that the subjects have established good rapport with their medical acquaintances despite the differences in their field of career. There is also a need to introduce dentistry as a compulsory unit in other health sciences, so as to improve knowledge of the profession and thus, improve the quality of the profession. This will improve equity and teamwork, hence improve management of patients.

\section{References:-}

1. Alysson, J., Dentist Vs. Doctor. Work.

2. Doctor of Medicine: Steps to Become a Medical Doctor.

3. Association, A.D., Glossary of Dental Clinical and Administrative Terms. 2014.

4. Suddick, R.P. and N.O. Harris, Historical perspectives of oral biology: a series. Critical reviews in oral biology and medicine: an official publication of the American Association of Oral Biologists, 1989. 1(2): p. 135-151.

5. Association, A.D., Dentists: Doctors of Oral Health. 2014.

6. Petersen, P.E., The World Oral Health Report 2003: continuous improvement of oral health in the 21st centurythe approach of the WHO Global Oral Health Programme. Community Dentistry and oral epidemiology, 2003. 31(s1): p. 3-24.

7. Lalla, E. and P.N. Papapanou, Diabetes mellitus and periodontitis: a tale of two common interrelated diseases. Nature Reviews Endocrinology, 2011. 7(12): p. 738-748.

8. Mays, J.W., M. Sarmadi, and N.M. Moutsopoulos, Oral manifestations of systemic autoimmune and inflammatory diseases: diagnosis and clinical management. Journal of Evidence Based Dental Practice, 2012. 12(3): p. 265-282.

9. Ranjitkar, S., R.J. Smales, and J.A. Kaidonis, Oral manifestations of gastroesophageal reflux disease. Journal of Gastroenterology and hepatology, 2012. 27(1): p. 21-27.

10. Wong, H., et al., Oral health-related quality of life in Hong Kong preschool children. Caries research, 2011. 45(4): p. 370-376.

11. Krol, D.M., Children's oral health and the role of the pediatrician. Current opinion in pediatrics, 2010. 22(6): $\mathrm{p}$. 804-808.

12. El-Solh, A.A., Association between pneumonia and oral care in nursing home residents. Lung, 2011. 189(3): p. 173-180.

13. Lam, O.L., et al., A systematic review of the effectiveness of oral health promotion activities among patients with cardiovascular disease. International journal of cardiology, 2011. 151(3): p. 261-267.

14. Akar, H., et al., Systemic consequences of poor oral health in chronic kidney disease patients. Clinical Journal of the American Society of Nephrology, 2011. 6(1): p. 218-226.

15. Migliorati, C. and C. Madrid, The interface between oral and systemic health: the need for more collaboration. Clinical Microbiology and Infection, 2007. 13(s4): p. 11-16. 